\title{
Frame Alignment Stability Issues in Natural Field Orientation
}

\author{
R.E. Betz ${ }^{*}$ and G. Mirzaeva ${ }^{\dagger}$ \\ School of Electrical Engineering and Computer Science \\ University of Newcastle, Callaghan, NSW, Australia, 2308 \\ Email: *Robert.Betz@newcastle.edu.au, †Galina.Mirzaeva@newcastle.edu.au
}

\begin{abstract}
Natural Field Orientation (NFO) is a technique for generating a rotating reference frame position for an induction machine which is aligned with the stator flux. The term "natural" is applied because there is an implicit tendency for the rotating frame to realign with the correct stator flux frame position if there is a perturbation away from this position. However, under regeneration conditions this realignment property may not occur, and the frame position, if perturbed, will move the control reference frame away from stator flux alignment. This paper examines this frame alignment stability problem, and proposes a solution that retains the essential simplicity of the NFO concept.
\end{abstract}

\section{INTRODUCTION}

Field oriented control (FOC) of induction machines is now a very mature area, with many commercial applications. Since its initial introduction by Blaschke [1], considerable research has taken place to refine the basic idea, and to overcome problems and issues related to practical implementation.

Much of the research in FOC techniques has concentrated on two main areas:

- Techniques for accurately locating the field position in induction machines. This is problematic as the required field positions cannot be directly measured from stator terminal measurements, and some estimation is required.

- Methods of implementing sensorless control of the machine. The terms "sensorless" in this context means that there is no shaft position sensor or speed transducer. Therefore field location information, as well as the shaft speed have to be determined without the use of this sensor.

Research into field position determination has largely concentrated on locating the rotor flux vector so that indirect field orientation can be used. The normal technique for doing this involves measuring the rotor speed, and then adding the slip frequency to give the angular velocity of the rotor flux, and finally integrating this to give the spatial location of the rotor flux. This location is the frame used for the control [2]. Unfortunately dependence on slip frequency implicitly makes the technique dependent on rotor resistance, which changes substantially during operation because of heating effects. Consequently the rotor flux position is erroneous, and performance is compromised.

Natural Field Orientation (NFO), is a patented concept that was first developed in the 1980s [3]. It is closely related to stator flux orientation. The essential idea behind NFO is that if one can sense the stator flux voltage, then assuming that the flux is at a constant value, the stator flux vector will be $90^{\circ}$ spatially lagging this voltage. One does not estimate the stator flux magnitude, as the strategy assumes that the flux is at the reference value. This is a crucial difference between NFO and stator flux orientation (SFO).

Remark 1: The last sentence of the previous paragraph is very important with respect to the NFO technique. If one assumes that the stator flux is at the reference level, then by using the magnitude of the stator flux voltage the angular velocity of the stator flux vector can be estimated. This angular velocity can then be integrated to give a less noisy estimate of the stator flux position in the machine compared to using the instantaneous value obtained from the stator flux voltage.

Remark 2: The stator flux voltage can be detected using the following expression:

$$
\underline{e}_{m s}=\underline{u}_{s}-R_{s} \underline{i}_{s}
$$

where $\underline{e}_{m s}$ is the stator flux voltage space vector, $\underline{u}_{s}$ is the input terminal voltage space vector, and $\underline{i}_{s}$ is the stator current space vector. Note that the estimation of $\underline{e}_{m s}$ only requires knowledge of the $R_{s}$ parameter, which is far fewer parameters than most other schemes.

A block diagram of the basic NFO scheme orientated to the stator flux vector ${ }^{1}$ is shown in Figure 1. Figure 2 shows the block diagram of a traditional SFO scheme. One can see both block diagrams are very similar, with the unique feature of NFO being that there is no integration to get the fluxes of the machine.

There have been a number of successful implementations of NFO control reported in the literature [4-6]. However, as shall be demonstrated in this paper, NFO has a fundamental static stability problem with respect to the location of the reference frame - i.e. if there is an error in the reference frame used for the control compared to the real reference frame position, then this error will tend to increase if the machine is in prolonged regeneration. If the machine is motoring then the "natural" field orientation will result in a realignment of the errant reference frame.

Remark 3: It should be noted that the stability problem being analysed here is a static stability problem. Therefore the

\footnotetext{
${ }^{1}$ One can also develop NFO in the rotor flux frame but this involves more parameter knowledge.
} 


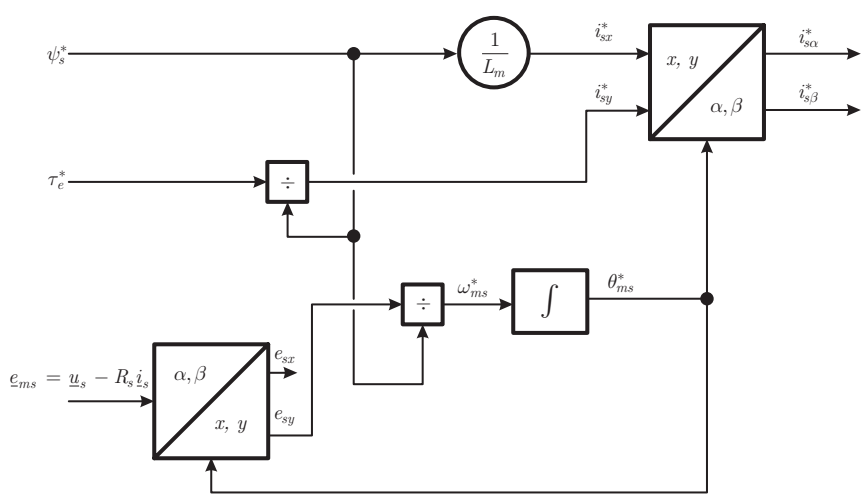

Fig. 1. Block diagram of a natural field orientation control system.

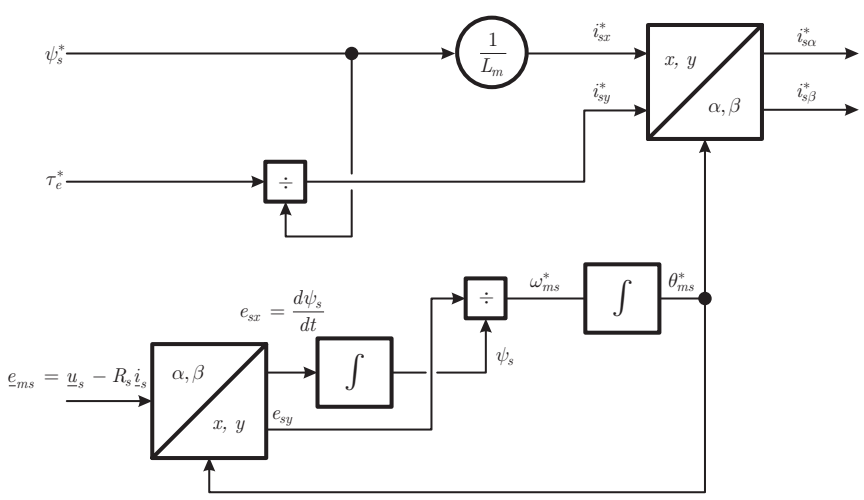

Fig. 2. Block diagram of a traditional stator flux oriented control system.

analysis in this paper does not take into account the dynamics of the system.

Remark 4: As far as the authors are aware the NFO frame stability problem demonstrated in this paper has not been previously published in the open literature.

This paper will concentrate on the current fed induction machine - i.e. both axes of the machine are driven by current sources. There is also a variant that uses a voltage drive in one axis of the machine and a current drive in the other axis [7-9], but this will not be considered here.

The remainder of this paper is organised as follows. Section II outlines the frame stability problem both heuristically and analytically. Section II-B will develop the solution approach to the stability problem, and then analyse and simulate it. Section III will present some preliminary experimental results of NFO with the stabilisation strategy applied. Finally Section IV will present the conclusions that can be drawn from this paper.

\section{The NFO Frame Stability Problem}

Before considering the formal analysis of the NFO frame alignment stability problem it would be beneficial to consider NFO frame alignment heuristically for both motoring and generating.

The following discussion is with reference to Figure 3. There are three axes on this figure - the $\alpha \beta$ axes are the axes for

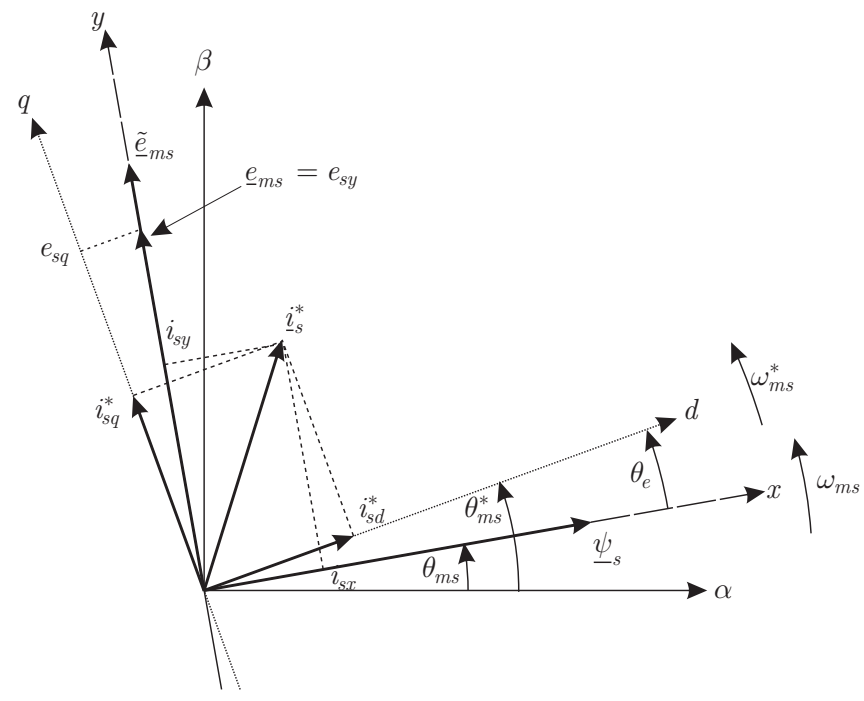

Fig. 3. Space vector diagram of an induction machine under motoring mode.

the stationary frame, the $d q$ axes are the axes for the estimated stator flux frame that the control is carried out in (the so-called control frame), and finally the $x y$ axes align with the actual stator flux axis. The angular velocity of the true stator flux vector (i.e. $x y$ frame) is $\omega_{m s}$, whereas the angular velocity of the control frame is $\omega_{m s}^{*}$.

From Figure 1 one can see that the basic equations for NFO can be written as:

$$
\begin{aligned}
& \omega_{m s}=\frac{e_{s y}}{L_{m}\left|\underline{i}_{m s}\right|}=\frac{e_{s y}}{\left|\underline{\psi}_{s}\right|} \\
& \omega_{m s}^{*}=\frac{e_{s q}}{L_{m}\left|\underline{i}_{m s}^{*}\right|}=\frac{e_{s q}}{\left|\underline{\psi}_{s}^{*}\right|} \\
& \tilde{\omega}_{m s}=\frac{\left|\underline{\tilde{e}}_{m s}\right|}{\left|\underline{\psi}_{s}^{*}\right|}
\end{aligned}
$$

and the frame position is:

$$
\theta_{m s}^{*}=\int \omega_{m s}^{*} d t+\theta_{m s_{\text {init }}}^{*}
$$

The back-emf vector $\underline{\underline{\tilde{e}}}_{m s}$ is the back-emf produced if the reference flux in the machine is the actual flux in the machine. Therefore $\tilde{\omega}_{m s}$ is the angular velocity of the reference frame if the flux in the machine is at the reference flux level of $\left|\underline{\psi}_{s}^{*}\right|$.

If everything is precise and ideal then $\omega_{m s}=\omega_{m s}^{*}=\tilde{\omega}_{m s}^{s}$, and $\theta_{s}^{*}=\theta_{s}$ (the true frame position). However if a frame error has occurred, then the situation of Figure 3 may arise. In this particular case the control frame is ahead of the $x y$ frame. Consequently the projections of the currents will be incorrect on the true $x y$ frame.

Under the misalignment condition of Figure 3 the actual back-emf $\underline{e}_{m s}$ is less than $\underline{\tilde{e}}_{m s}$ since the flux producing current is the projection of $i_{s d}^{*}$ as $i_{s x}$ onto the $x y$ axis. Therefore $i_{s x}<i_{s d}^{*}$ and $\left|\underline{\psi}_{s}\right|<\left|\underline{\psi}_{s}^{*}\right|$. Consequently the projection of $\underline{e}_{m s}$ as $e_{s q}$ onto the $d q$ control axes means that $e_{s q}$ is even smaller than it would otherwise be under correct alignment. This means from (3) that $\omega_{m s}^{*}$ is less than both $\omega_{m s}$ and $\tilde{\omega}_{m s}$, 


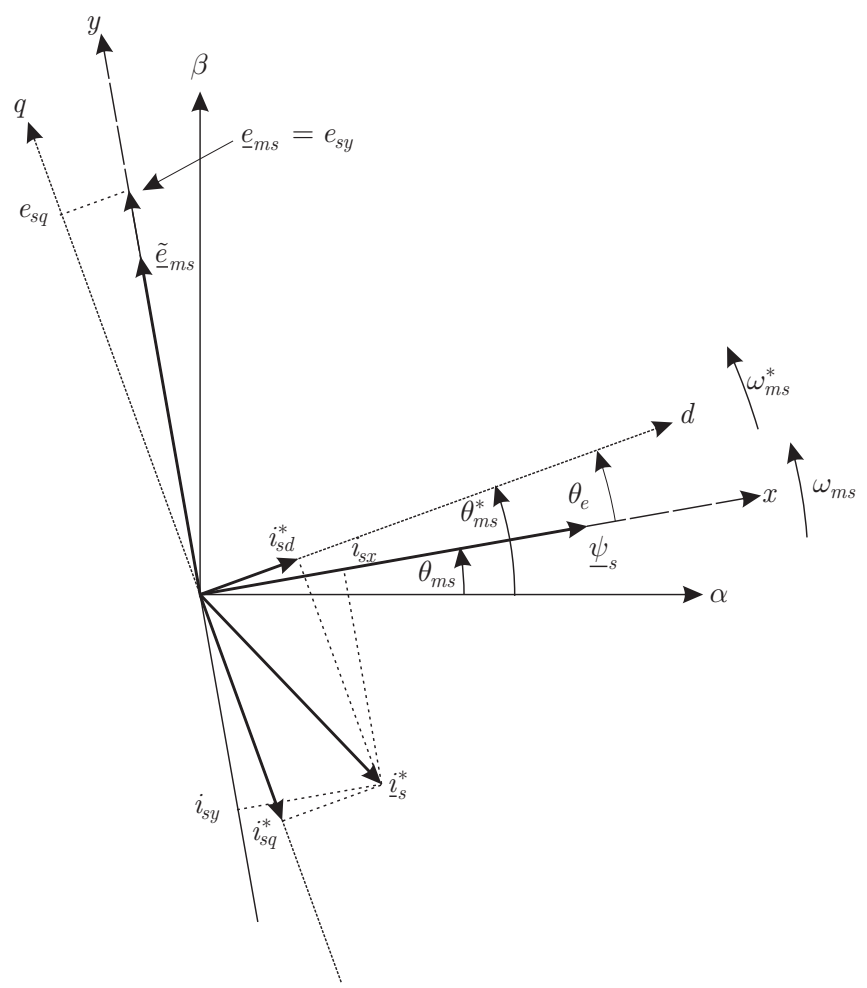

Fig. 4. Space vector diagram of induction machine under regeneration.

and therefore the $d q$ frame will rotate more slowly than the exact frame would, and hence under the integration of (5) the control frame will approach the exact frame. This also means that the $\theta_{e} \rightarrow 0$ and the $d q$ and $x y$ frames approach each other.

Remark 5: Note that the $x y$ frame does not necessarily rotate at the correct frame angular velocity $\tilde{\omega}_{m s}$, but under this effect $\omega_{m s} \rightarrow \tilde{\omega}_{m s}$ as well. Therefore the $x y$ and $d q$ frames realign, and the original misalignment has been naturally corrected, hence the name of the frame alignment technique. The same argument applies if the $\theta_{e}$ error is such that $d q$ lags behind $x y$, and for the other rotation direction.

Now consider the situation under regeneration. A similar argument can be mounted using Figure 4, which shows the same situation as Figure 3 except that the machine is regenerating. In this case the $i_{s x}$ projection value is larger than the $i_{s d}^{*}$ and hence $\left|\underline{e}_{m s}\right|>\left|\underline{\tilde{e}}_{m s}\right|$, which results in:

$$
\omega_{m s}^{*}=\frac{e_{s q}}{\left|\underline{\psi}_{s}^{*}\right|}>\tilde{\omega}_{m s}=\frac{\left|\underline{\tilde{e}}_{m s}\right|}{\left|\underline{\psi}_{s}^{*}\right|}
$$

i.e. the $d q$ frame will rotate faster than the $x y$ frame under correct alignment. Under misalignment condition though the exact frame rotates at $\omega_{m s}$ different from $\tilde{\omega}_{m s}$, so two situations are possible. If $\omega_{m s}^{*}<\omega_{m s}$ then regeneration is stable. If $\omega_{m s}^{*}>\omega_{m s}$ (the more likely condition) then $d q$ and $x y$ frames in Figure 4 will move apart - i.e. $\theta_{e}$ will increase. Under typical conditions in regeneration mode the frame alignment error will grow and therefore the frame alignment is unstable.

In order to verify this instability a number of simulation studies were carried out using the Saber ${ }^{\circledR}$ simulation package.

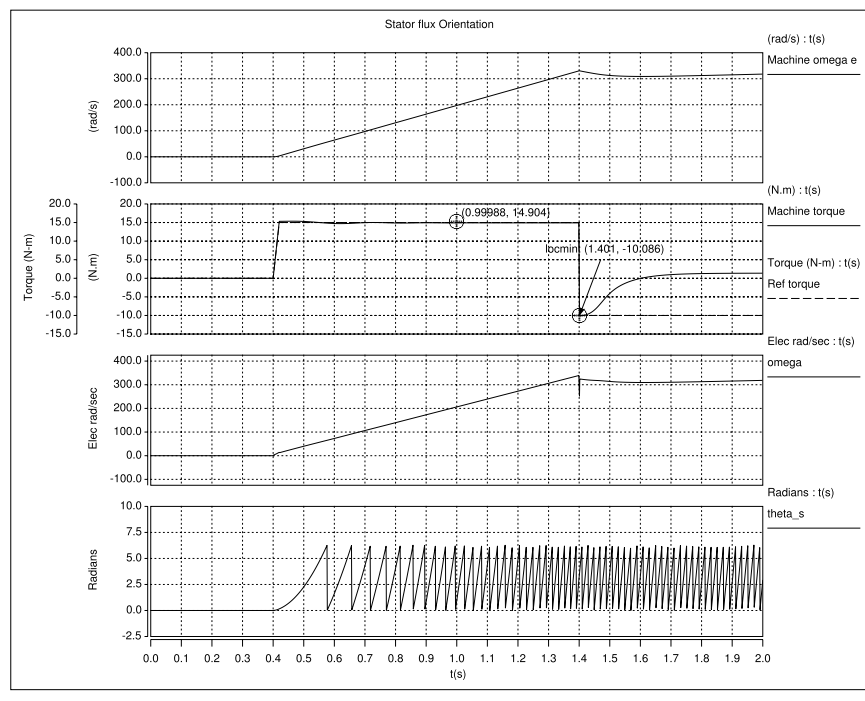

Fig. 5. Motoring and regeneration using NFO without stabilising control.

The simulations were carried out using the standard NFO algorithm with all parameters known and ideal currents sources to model a current controlled voltage source inverter. There was no switching in the simulation. Therefore the simulation is very idealised. Figure 5 shows one of these simulation results and illustrates the effect of frame instability in regeneration. Notice that NFO works correctly in motoring mode, but as soon as regeneration starts the frame alignment is lost and the torque output diverges from the correct value.

\section{A. Stability analysis}

In order to generate the operational quadrants where the frame alignment is stable and unstable we shall consider the rate of change of the frame position error:

$$
\frac{d \theta_{e}}{d t}=\omega_{m s}^{*}-\omega_{m s}
$$

where $\theta_{e}=\theta_{m s}^{*}-\theta_{m s}$. For the stability of the frame position we require $\frac{1}{\theta_{e}} \frac{d \theta_{e}}{d t}<0$. In order to develop the expression we utilise the fact that $i_{s d}^{*}$ and $i_{s q}^{*}$ are independent of the frame position (as can be deduced from Figure 1).

One can write the following expression for $\omega_{m s}^{*}$ :

$$
\omega_{m s}^{*}=\frac{e_{s q}}{L_{m}\left|\underline{i}_{m s}^{*}\right|}=\frac{e_{s y} \cos \theta_{e}}{\left|\underline{\psi}_{s}^{*}\right|}=\frac{\left|\underline{\psi}_{s}\right| \cos \theta_{e}}{\left|\underline{\psi}_{s}^{*}\right|} \omega_{m s}
$$

We can also write the following for the actual flux in the machine in terms of the reference currents:

$$
\left|\underline{\psi}_{s}\right|=L_{m}\left|\underline{i}_{m s}\right|=L_{m} i_{s x}=L_{m}\left(i_{s d}^{*} \cos \theta_{e}-i_{s q}^{*} \sin \theta_{e}\right)
$$

Using (9) and (8) we can write:

$$
\omega_{m s}^{*}=\left(\cos ^{2} \theta_{e}-\frac{i_{s q}^{*}}{i_{s d}^{*}} \sin \theta_{e} \cos \theta_{e}\right) \omega_{m s}
$$

It is possible to manipulate the induction machine equations in a stator flux reference frame, ignoring the leakage inductance, into the following form [10]:

$$
L_{m} \frac{d\left|\underline{i}_{m s}\right|}{d t}=R_{r}\left(\underline{i}_{s \psi s}-\left|\underline{i}_{m s}\right|\right)-j\left(\omega_{m s}-\omega_{r}\right)\left|\underline{i}_{m s}\right| L_{m}
$$


which can be separated into real and imaginary components to give:

$$
\begin{aligned}
L_{m} \frac{d\left|\underline{i}_{m s}\right|}{d t} & =R_{r}\left(i_{s x}-\left|\underline{i}_{m s}\right|\right) \\
0 & =R_{r} i_{s y}-\left(\omega_{m s}-\omega_{r}\right)\left|\underline{i}_{m s}\right| L_{m}
\end{aligned}
$$

Remark 6: Note from (12) one can see that if the stator flux is in steady state then the stator current is the stator magnetising current of the machine. Equation (13) is very similar to the equivalent expression for FOC [2].

Using equations (13) and (9), as well as $i_{s y}=i_{s d}^{*} \sin \theta_{e}+$ $i_{s q}^{*} \cos \theta_{e}$, and substituting into (10) allows us to write:

$$
\omega_{m s}=\frac{R_{r}\left(i_{s d}^{*} \sin \theta_{e}+i_{s q}^{*} \cos \theta_{e}\right)}{L_{m}\left(i_{s d}^{*} \cos \theta_{e}-i_{s q}^{*} \sin \theta_{e}\right)}+\omega_{r}
$$

Substituting (14) and (10) into (7) (assuming no leakage) one can get:

$$
\begin{array}{r}
\frac{d \theta_{e}}{d t}=\left(\frac{\left(i_{s d}^{*} \cos \theta_{e}-i_{s q}^{*} \sin \theta_{e}\right) \cos \theta_{e}}{i_{s d}^{*}}-1\right) \\
\left(\frac{R_{r}\left(i_{s d}^{*} \sin \theta_{e}+i_{s q}^{*} \cos \theta_{e}\right)}{L_{m}\left(i_{s d}^{*} \cos \theta_{e}-i_{s q}^{*} \sin \theta_{e}\right)}+\omega_{r}\right)
\end{array}
$$

Remark 7: It is very difficult to gain any insight from (15) due to its highly non-linear nature. Therefore, assuming $\theta_{e}$ is small, the following approximations can be made to simplify the expression:

$$
\begin{aligned}
\cos \theta_{e} & \approx 1 \\
\sin \theta_{e} & \approx \theta_{e} \\
i_{s d}^{*} i_{s q}^{*} \theta_{e} & \ll\left(i_{s d}^{*}\right)^{2} \text { and }\left(i_{s q}^{*}\right)^{2}
\end{aligned}
$$

Using the approximations in Remark 7 one can write (15) as:

$$
\frac{d \theta_{e}}{d t} \approx-\left[\frac{R_{r}}{L_{m}}\left(\frac{i_{s q}^{*}}{i_{s d}^{*}}\right)^{2}+\left(\frac{\omega_{r} i_{s q}^{*}}{i_{s d}^{*}}\right)\right] \theta_{e}
$$

which can be written as:

$$
\frac{d \theta_{e}}{d t}=-\left[\frac{1}{T_{r m}} x^{2}+\omega_{r} x\right] \theta_{e}=-x\left[\frac{1}{T_{r m}} x+\omega_{r}\right] \theta_{e}
$$

where $x=i_{s q}^{*} / i_{s d}^{*}$ and $T_{r m}=L_{m} / R_{r}$

For stability we require $\frac{1}{\theta_{e}} \frac{d \theta_{e}}{d t}<0$, which means that:

$$
x\left[\frac{1}{T_{r m}} x+\omega_{r}\right]>0
$$

or

$$
\operatorname{sign}(x)=\operatorname{sign}\left(\frac{1}{T_{r m}} x+\omega_{r}\right)
$$

In motoring mode (assuming $\omega_{r}>0$ ), and realising that $x>$ 0 then equation (22) is always satisfied. Therefore motoring is always stable supporting the previous heuristic reasoning.

Now consider regeneration. In this case (again assuming $\omega_{r}>0$ ), with $x<0$ (since $i_{s q}^{*}<0$ to give negative torque), then:

$$
\frac{1}{T_{r m}} x+\omega_{r}<0 \Rightarrow x<-\omega_{r} T_{r m}
$$

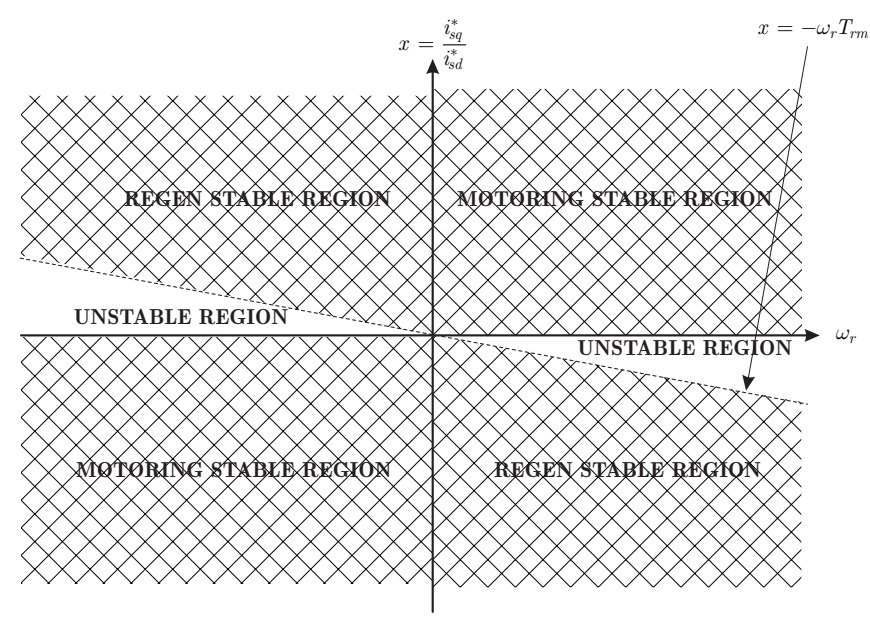

Fig. 6. Stable/unstable areas for uncompensated NFO.

\begin{tabular}{|l|c|c|c|c|}
\hline Mode & $\omega_{\mathbf{r}}$ & $\mathbf{i}_{\mathbf{s q}}^{*} / \mathbf{i}_{\mathbf{s d}}^{*}(\mathbf{x})$ & $\theta_{\mathbf{e}}$ & Condition \\
\hline \hline Motoring & $>0$ & $>0$ & $>0$ & $x>-\omega_{r} T_{r m}$ \\
\cline { 2 - 5 } & $>0$ & $>0$ & $<0$ & $x>-\omega_{r} T_{r m}$ \\
\cline { 2 - 5 } & $<0$ & $<0$ & $>0$ & $x<-\omega_{r} T_{r m}$ \\
\cline { 2 - 5 } Generating & $>0$ & $<0$ & $<0$ & $x<-\omega_{r} T_{r m}$ \\
\cline { 2 - 5 } & $>0$ & $<0$ & $>0$ & $x<-\omega_{r} T_{r m}$ \\
\cline { 2 - 5 } & $>0$ & $<0$ & $<0$ & $x<-\omega_{r} T_{r m}$ \\
\cline { 2 - 5 } & $<0$ & $>0$ & $>0$ & $x>-\omega_{r} T_{r m}$ \\
\cline { 2 - 5 } & $<0$ & $<0$ & $x>-\omega_{r} T_{r m}$ \\
\hline
\end{tabular}

TABLE I

SUMMARY OF STABILITY BOUNDARY CONDITIONS FOR MOTORING AND GENERATING.

for (22) to be satisfied.

If the stability regions for both motoring and regeneration are plotted on a $\omega_{r}$ versus $x$ plot then Figure 6 results. The unstable regions are a significant part of the operational envelope in regeneration mode.

The results for both motoring and regeneration are tabulated in Table I. The areas of crosshatching correspond to the areas that satisfy the $x$ criteria and the "Condition" criteria. For example, for motoring with $\omega_{r}>0$ and $\theta_{e}>0$, then the region where $d \theta_{e} / d t<0$ is where the regions defined by $x>0$ and $x>-\omega_{r} T_{r m}$ overlap - i.e. the 1 st quadrant in Figure 6. Similar arguments can be made for other areas in Figure 6.

Remark 8: The vertical scale in Figure 6 is deceptive in that it makes the unstable region look small. On a $\tau_{e}$ versus $\omega_{r}$ scale this region is much larger than it appears in Figure 6.

In order to check the validity of the linearised form of this analysis the full non-linear expression was plotted using a $3 \mathrm{~d}$ plotting tool. Two cases were plotted - one for motoring and one for regeneration, and these appear a Figures 7 and 8 respectively. As an example of the interpretation of these figures consider Figure 7. For $\theta_{e}<0$ then $d \theta_{e} / d t>0$ indicating that the frame is catching up and $\left|\theta_{e}\right|$ will get smaller. For $\theta_{e}>0$ then $d \theta_{e} / d t<0$ indicating that the frame angle error is decreasing forcing the frames to align.

Remark 9: In Figure 7 one can see, based on the reasoning 
Non-linear, No leakage, Motoring, $\omega_{r}=20, \tau_{e}>0$

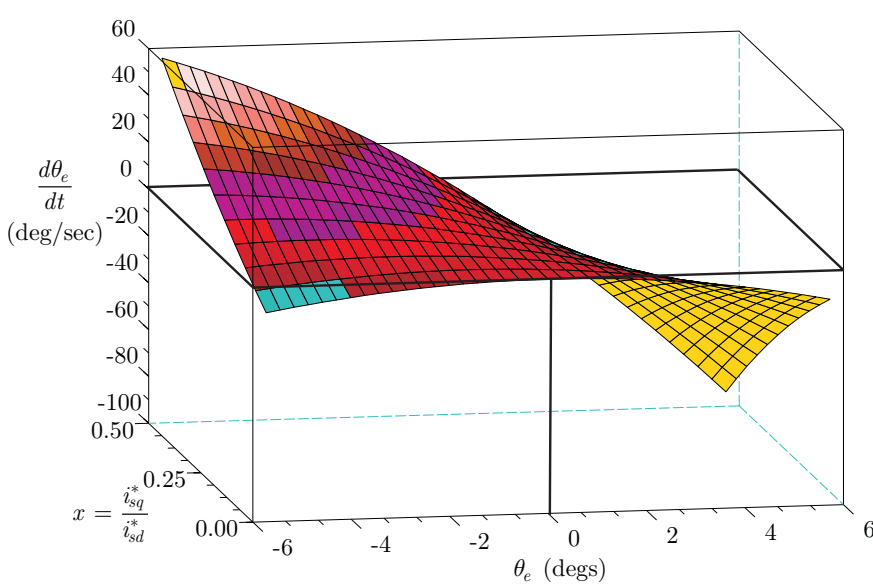

Fig. 7. Plot of equation (15) for motoring, $\omega_{r}=20 \mathrm{r} / \mathrm{s}$ (No Leakage).

Non-linear, Regeneration, $\omega_{r}=20 \mathrm{r} / \mathrm{s}$

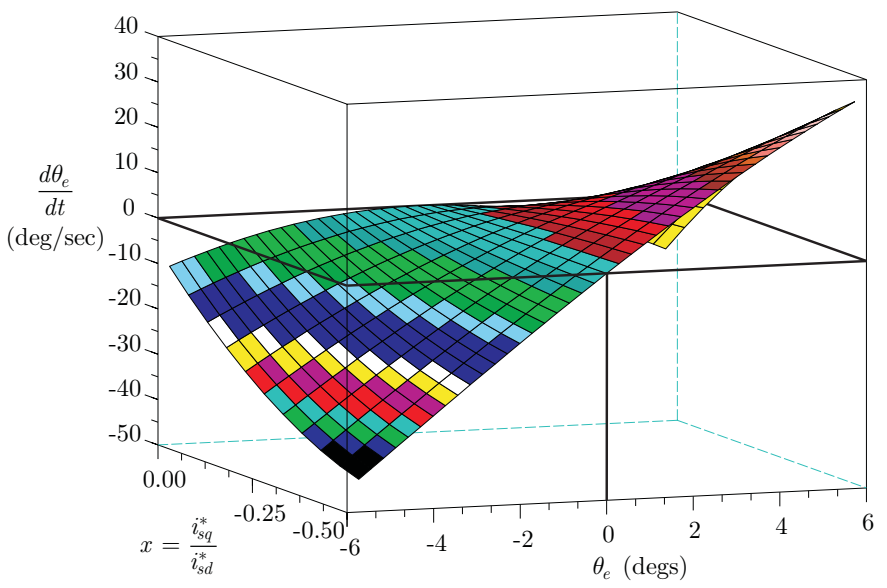

Fig. 8. Plot of equation (15) for regeneration, $\omega_{r}=20 \mathrm{r} / \mathrm{s}$ (No Leakage)

in the paragraph above, that for small $x$ (i.e. small torques) and $\theta_{e}<0$ that $d \theta_{e} / d t<0$ meaning that the frame will move away. This did not show up in the linearised analysis. Therefore, even under motoring there are conditions where NFO displays static frame position instability.

Examination of Figure 8 for regeneration shows, as predicted from the linearised analysis, that the frame alignment is statically unstable in most regions of operation. There is similarity with the motoring case, in that at very low torques (i.e. $x$ small) the regeneration mode is stable for $\theta_{e}>0$.

Remark 10: Figures 7 and 8 indicate that the linearised analysis is broadly representative of the performance of the system when leakage inductance effects are ignored in the machine.

\section{B. Stabilisation Strategy}

Examination of Figures 3 and 4 indicates that when misalignment of the $d q$ and $x y$ axes occurs then an $e_{s d}$ voltage will appear. The presence of this signal can be used to provide

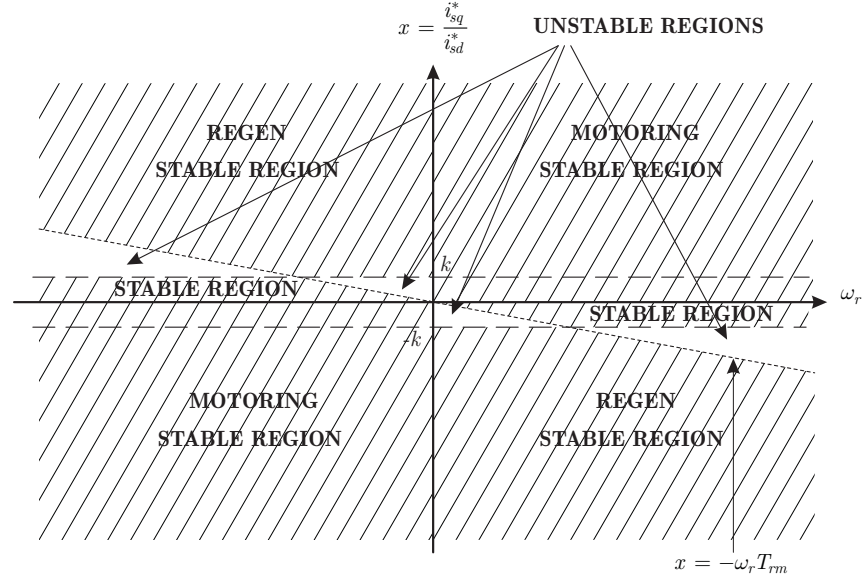

Fig. 9. Stable/unstable regions for NFO with compensating $e_{s d}$ feedback.

auxiliary feedback that can stabilise the instability identified in Section II-A. Based on the heuristic reasoning used in this section one can develop the following augmentation to the frame angular velocity expression:

$$
\omega_{m s}^{*}=\frac{e_{s q}-\operatorname{sign}\left(e_{s q}\right) k e_{s d}}{\left|\underline{\psi}_{s}^{*}\right|}
$$

where $k$ is included as a tunable gain parameter.

Using this expression stability analysis similar to that for the uncompensated NFO can be carried out. Due to the similarity of the manipulations to the previous one we shall simply present the resulting expression [10]:

$$
\begin{aligned}
\frac{d \theta_{e}}{d t}=[ & \cos ^{2} \theta_{e}+\operatorname{sign}\left(e_{s q}\right) k \frac{i_{s q}^{*}}{i_{s d}^{*}} \sin ^{2} \theta_{e} \\
& \left.-\left(\frac{i_{s q}^{*}}{i_{s d}^{*}}+\operatorname{sign}\left(e_{s q}\right) k\right) \cos \theta_{e} \sin \theta_{e}-1\right] \\
& {\left[\frac{R_{r}}{L_{m}}\left(\frac{i_{s d}^{*} \sin \theta_{e}+i_{s q}^{*} \cos \theta_{e}}{i_{s d}^{*} \cos \theta_{e}-i_{s q}^{*} \sin \theta_{e}}\right)+\omega_{r}\right] }
\end{aligned}
$$

which can be simplified using similar approximations as those of Remark 7 to:

$$
\frac{d \theta_{e}}{d t} \approx-\left(\frac{i_{s q}^{*}}{i_{s d}^{*}}+\operatorname{sign}\left(e_{s q}\right) k\right)\left(\frac{R_{r}}{L_{m}} \frac{i_{s q}^{*}}{i_{s d}^{*}}+\omega_{r}\right) \theta_{e}
$$

which can be written as (with $x$ and $T_{r m}$ as defined previously):

$$
\frac{d \theta_{e}}{d t} \approx-\left(\operatorname{sign}\left(e_{s q}\right) k+x\right)\left(\frac{1}{T_{r m}} x+\omega_{r}\right) \theta_{e}
$$

Remark 11: Note that if $k=0$ in (27) then the expression collapses to (20).

If similar logic is applied to (27) as was done with (20) then the stable and unstable areas of Figure 9 result.

Remark 12: One can see from Figure 9 that the unstable regions have not been completely eliminated. However the unstable areas have been decreased. Furthermore, by dynamically changing the feedback term $k$ one can change the 


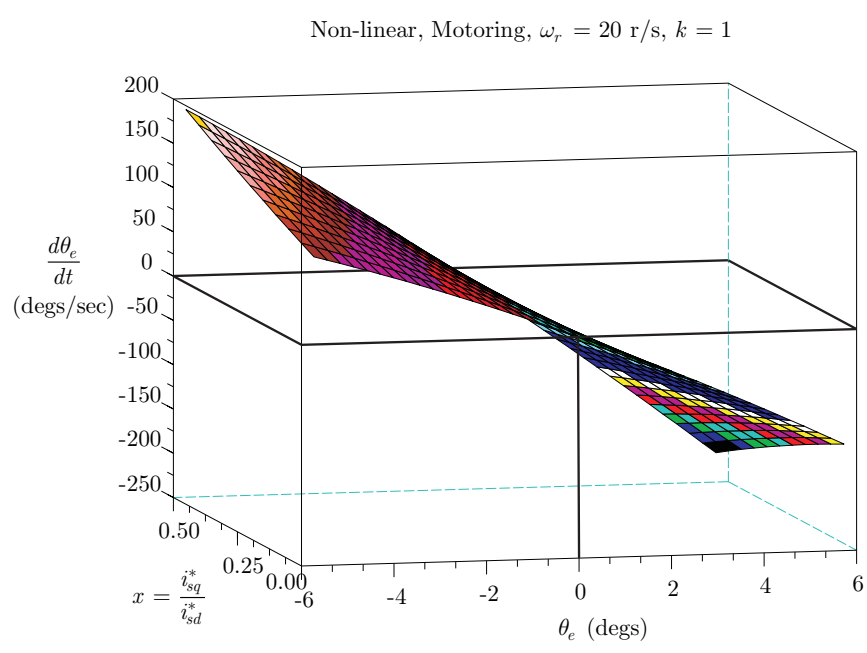

Fig. 10. Plot of equation (25) under motoring with feedback $k=1$, (No Leakage)

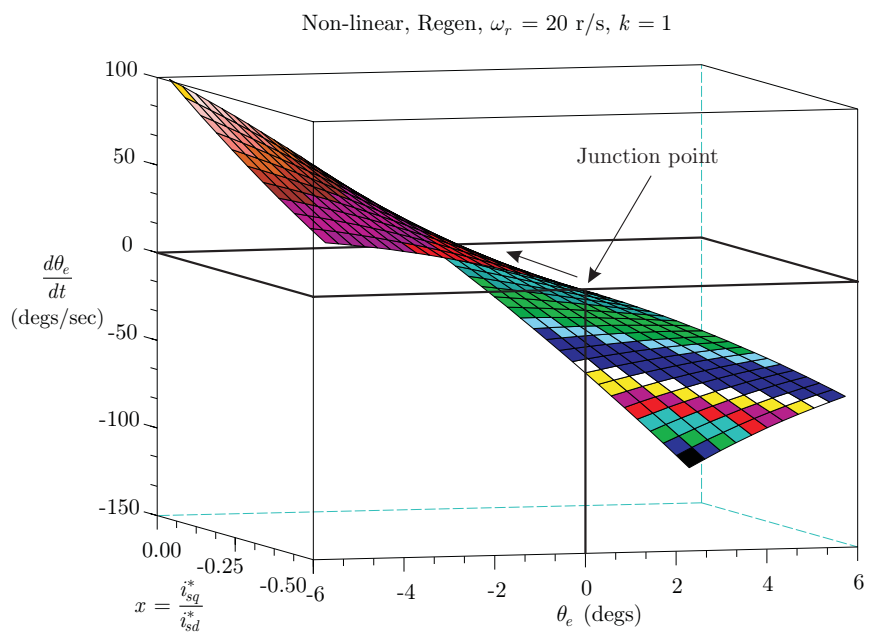

Fig. 11. Plot of equation (25) under regeneraton with feedback $k=1$, (No Leakage)

location of the stable/unstable regions so that stability can be guaranteed.

As with the un-augmented NFO case the full non-linear expression represented by equation (25) has been plotted for both the motoring and regeneration cases. The feedback gain used in these plots is $k=1$. As with the previous similar plots the angular velocity of the machine is arbitrarily chosen to be $20 \mathrm{radians} / \mathrm{sec}$ (which is also representative of what happens at other speeds).

Figure 10 shows the situation when motoring. One can see that the whole motoring region is now stable, and the small area of static instability observed in the non-feeback case shown in Figure 7 is no longer present.

Figure 11 is the plot of $d \theta_{e} / d t$ for regeneration with the feedback augmentation. One can see that the plot now resembles that for the motoring case, which means that it is now statically stable as predicted by the previous linearised

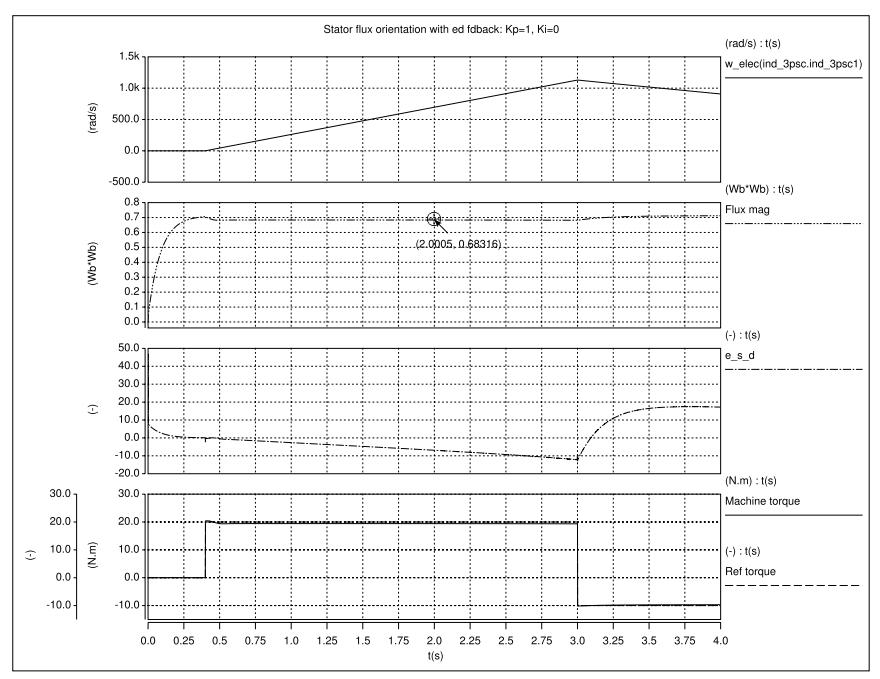

Fig. 12. Simulation of NFO algorithm with $e_{s d}$ feedback.

analysis.

Closer examination of Figure 11 gives a hint of the small regions of static instability shown in the linearised Figure 9. As one moves into the page from the junction point marked on the plot one can see that the shape bends to the left in the figure, hinting for negative $\theta_{e}$ that $d \theta_{e} / d t$ is also negative - i.e. static instability. Note that this situation occurs for relatively low values of torque (i.e. $x$ ).

Remark 13: The non-linear plots confirm that the linearised analysis results are very close to exact, and therefore are valid.

Simulation studies were conducted using the proposed stabilisation strategy. Figure 12 is representative of the results obtained. As can be seen the previously unstable regeneration region is now stable, and the torque performance of the system is good.

Remark 14: Note in Figure 12 that $e_{s d} \neq 0$, but relative to the value of $e_{s q}$ at the high shaft angular velocities in the simulation it is very small indicating that there is good frame alignment.

Remark 15: During the simulation studies it was observed that even the augmented NFO algorithm could still tend to misalign, especially when rapid and large torque transients were requested.

\section{EXPERIMENTAL RESUlts}

The experiments were carried out using a $38 \mathrm{~kW}$ IGBT based inverter connected to a $7.5 \mathrm{~kW}$ wye connected induction machine. This machine was mounted on a dynamometer test bed with a DC load machine configured as a simple Ward-Leonard system to provide static loading capable of regeneration or motoring operation.

The software used for the tests was a modification of existing software that implemented either field oriented or instantaneous power control algorithms. Therefore the underlying code for current control, sampling, and the basic timing could all 


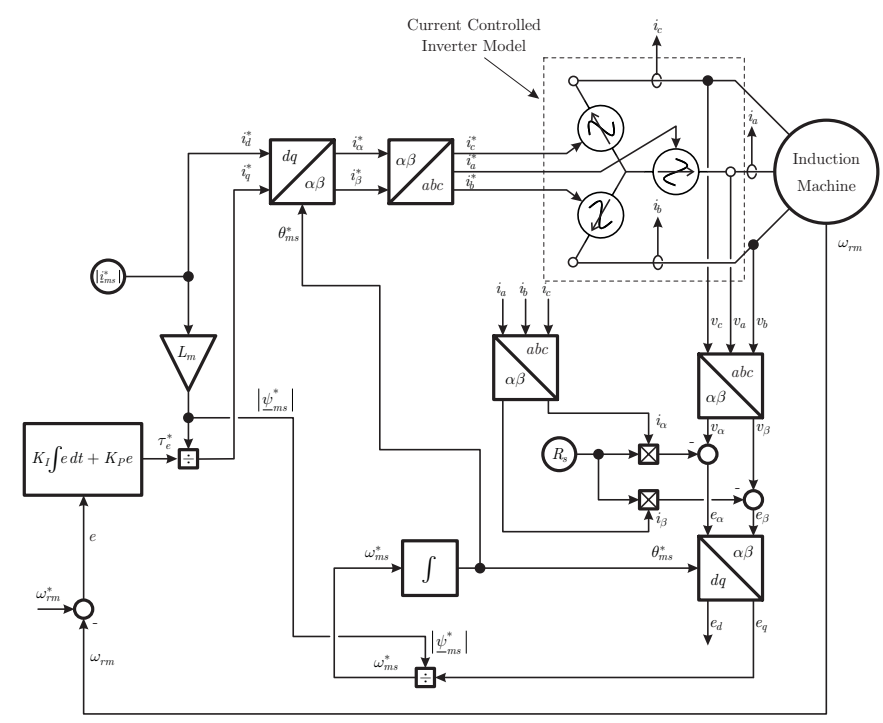

Fig. 13. Block diagram of the implementation of the basic NFO control on the experimental system.

be used. Only minor modifications were required for the NFO algorithm.

The code itself was written in ' $C$ ' and was executed in a TMS320C6701 floating point DSP. The control frequency was $3906.25 \mathrm{~Hz}$ corresponding to a control period of $256 \mu$ secs. Symmetrical PWM generation was used, therefore the current sampling occurred at the beginning and middle of each of the control intervals. The software was designed with an integrated data logging system allowing both internal control variables as well as sampled currents, voltages, and shaft speeds to be logged. The number of simultaneous variables that could be logged depends on the size of the logging buffers, and in most circumstances approximately 6 variables can be simultaneously logged for approximately 2 seconds for each variable. It is possible to trigger logging at a particular time, or to continuously log into a circular buffer.

Both normal (or basic) NFO algorithm, as well as the new proposed augmented NFO algorithm were implemented on the experimental system. Figure 13 shows the structure of the algorithm for the normal NFO with a PI speed control loop around it. Similarly the diagram for the augmented NFO controller appears in Figure 14.

All the experiments were conducted in steady state at a shaft of $75 \mathrm{rad} / \mathrm{sec}$ (mechanical). Basic NFO algorithm $(k=0)$ and the proposed augmented NFO algorithm with $k=1$ and $k=2$ were tested under a range of conditions. These included: no-load motoring test (4.5 $\mathrm{Nm}$ on the shaft due to losses), motoring at $20 \mathrm{Nm}$ and regeneration at $-6.7 \mathrm{Nm}$ of torque on the shaft. In addition, for each control algorithm the machine was statically loaded in regeneration mode up to its stability limit. The reference magnetising current $i_{s d}^{*}$ was always kept constant and equal to $10 \mathrm{Amp}$.

The following variables were simultaneously logged: $e_{s \alpha}$, $e_{s \beta}$ - projections of the stator flux voltage vector onto the

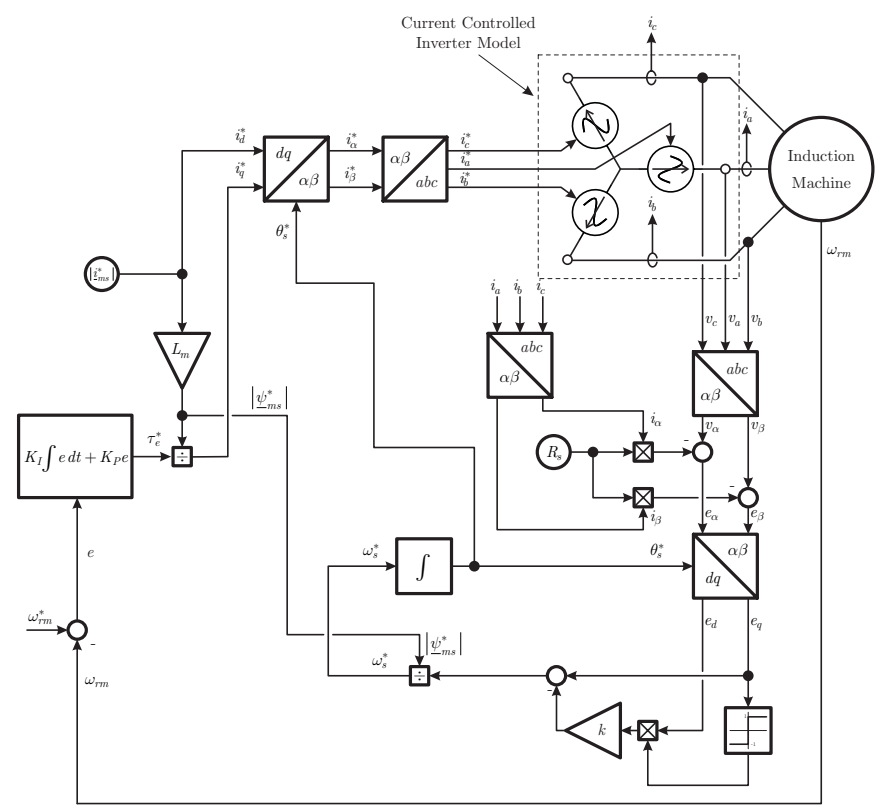

Fig. 14. Block diagram of the implementation of the $e_{s d}$ augmented NFO control on the experimental system.

\begin{tabular}{|c|c|c|c|c|c|c|}
\hline \multirow{2}{*}{ Torque } & \multicolumn{2}{|c|}{$\mathbf{k}=\mathbf{0}$} & \multicolumn{2}{c|}{$\mathbf{k}=\mathbf{1}$} & \multicolumn{2}{c|}{$\mathbf{k}=\mathbf{2}$} \\
\cline { 2 - 7 } & $\theta_{e}(\mathrm{deg})$ & $\mathbf{x}$ & $\theta_{e}(\mathrm{deg})$ & $\mathbf{x}$ & $\theta_{e}(\mathrm{deg})$ & $\mathbf{x}$ \\
\hline \hline $4.5 \mathrm{Nm}$ & 13.09 & -0.05 & 1.72 & 0.14 & 1.01 & 0.16 \\
\hline $4.5 \mathrm{Nm}$ & 35.32 & -0.48 & 6.6 & 0.06 & 3.53 & 0.11 \\
$0.9 L_{m}$ & & & & & & \\
\hline $20.0 \mathrm{Nm}$ & 7.14 & 0.36 & 0.99 & 0.80 & 0.64 & 0.78 \\
\hline$-6.7 \mathrm{Nm}$ & N/A & N/A & -4.93 & -0.12 & -2.65 & -0.19 \\
\hline
\end{tabular}

TABLE II

SUMMARY OF THE RESUlTS OF STEADY STATE TESTS FOR THE BASIC $(\mathbf{k}=\mathbf{0})$ AND the Augmented $(\mathbf{k}=\mathbf{1})$ And $(\mathbf{k}=\mathbf{2})$ NFO ALgorithms.

stationary frame axes; $e_{s d}, e_{s q}, i_{s q}^{*}$, and the mechanical speed of the shaft $\omega_{r m}$. From these measurements the following parameters were derived: the exact frame position $\theta_{m s}$ based on orientation of the stator flux voltage vector in the stationary frame; the control frame position $\theta_{m s}^{*}$ as the angle that converts $e_{s \alpha}, e_{s \beta}$ into $e_{s d}, e_{s q}$; the misalignment angle $\theta_{e}=\theta_{m s}^{*}-\theta_{m s}$; and $x=i_{s q}^{*} / i_{s d}^{*}$.

The results of the steady state tests are summarised in Table II. The basic NFO algorithm, despite being able to drive the machine to the required speed in motoring mode, demonstrated very poor frame alignment. When motoring at $4.5 \mathrm{Nm}$ the control frame was $13^{\circ}$ ahead of the exact frame. Because of this misalignment, the algorithm was seeing the torque producing current as being negative. With $20 \mathrm{Nm}$ on the shaft the frame alignment improved somewhat - down to $7^{\circ}$. We were not able to get the basic algorithm to work in generation mode at this speed - as the power flow direction was changing, the algorithm completely lost control, as illustrated in Figure 15.

Remark 16: Another observation with regard to the basic NFO algorithm was that it turned to be very sensitive to the 


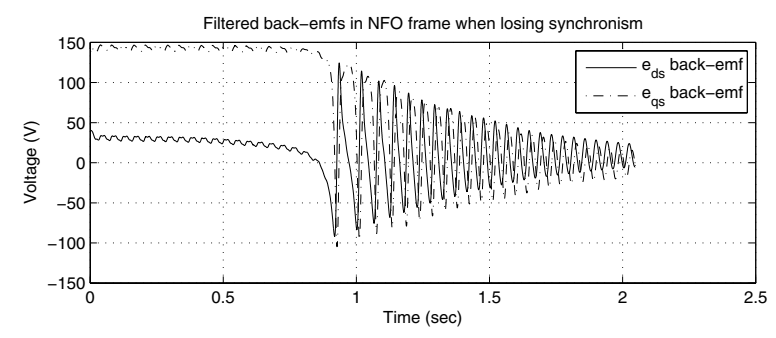

Fig. 15. Loss of synchronism when generating with the Basic NFO.
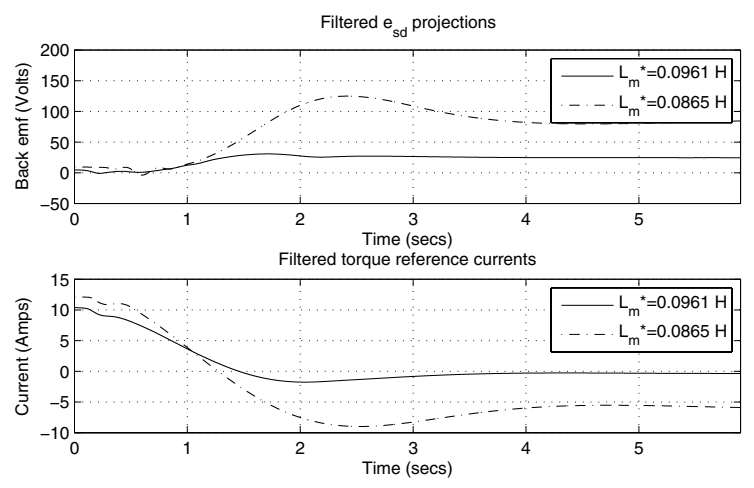

Fig. 16. Start-up plots for exact and $-10 \%$ erroneous $L_{m}$ values.

value of $L_{m}$. Sensitivity to the $R_{s}$ value was also observed, but to a much smaller degree.

As can be seen from the second row of the Table II, a $-10 \%$ error in $L_{m}$ caused the frame misalignment to increase from $13^{\circ}$ to $35^{\circ}$, and a small positive torque on the shaft was perceived as a high negative torque by the NFO algorithm. The tendency of the control frame to stabilise at some misaligned position with respect to that of the exact frame is illustrated by the start-up plots in Figure 16 that correspond to the motoring mode at $4.5 \mathrm{Nm}$ of torque. One can see that initially the control algorithm starts from almost zero values of $e_{s d}$, which indicates that there is no initial misalignment. But then $e_{s d}$ drifts away towards positive values and stabilises at some level that translates into positive $\theta_{e}$. Figure 16 also shows how torque producing current as seen by NFO floats from initially positive to negative values. By adjusting the $L_{m}$ value we were able to reduce the frame misalignment, but even in the best case for the given conditions the control frame was statically $10^{\circ}$ ahead of the exact frame.

The proposed augmentation of the NFO algorithm significantly improved its performance and frame alignment stability. With reference to the Table II, for $k=1$ the static frame misalignment in both motoring scenarios reduced to less than $2^{\circ}$, and for $k=2$ to even smaller values. In both cases the algorithm was able to control regeneration stably at $-6.7 \mathrm{Nm}$, though in this case negative deviations of the $e_{s d}$ values were observed. By further increasing negative torque we pushed the algorithm to its static stability limit. The stability torque limits were: for $k=1$, torque $=-12 \mathrm{Nm}$; and for $k=2$, torque $=-16 \mathrm{Nm}$. Synchronism was lost in a similar manner to that shown in Figure 15, except that $e_{s d}$ at the moment just before oscillations started had negative values in both cases. The augmentation significantly improved the algorithm, but it is clear that NFO stability issues need further investigation, both theoretically and experimentally.

\section{CONCLUSIONS}

The main conclusions that can be drawn from the results in this paper are:

- The "normal" NFO algorithm has a frame angle static instability for most of the regeneration region. This has been proved using a linearised analysis, and also from non-linear $3 \mathrm{~d}$ plots of $d \theta_{e} / d t$.

- The regeneration mode of operation can be made statically stable by augmenting the basic NFO algorithm with $\operatorname{sign}\left(e_{s q}\right) \times e_{s d}$ feedback. This has been proved to stabilise the static frame instability identified in the basic algorithm.

- Even with the stabilisation strategy NFO still displays, under some circumstances, a tendency to stabilise at a misaligned frame position, or to lose frame alignment completely. This is the subject of further investigation.

- The results of the analysis and simulation for both the basic and augmented NFO algorithms have been confirmed by experimental results. The experimental results confirm that with augmentation NFO is capable of sustaining both motoring and regeneration with "natural" field orientation still occurring.

Additional analysis of the system including the leakage inductance has indicated that it could have an important influence on the performance of the NFO algorithm. More detailed work is currently being carried out including the leakage, and will be the subject of a future paper.

\section{REFERENCES}

[1] F. Blaschke, "The principle of field-orientation as applied to the new TRANSVEKTOR closed-loop control system for rotating-field machines," Siemens Review, vol. 34, pp. 217-220, 1972.

[2] P. Vas, Vector Control of AC Machines. Oxford University Press, 1990, iSBN 0-19-859370-8.

[3] R. Jönsson, "Method and apparatus for controlling an AC motor," United States Patent 4,458,193, July 1984.

[4] R. Jönsson and W. Leonhard, "Control of an induction motor without a mechanical sensor, based on the principle of natural field orientation (NFO)," in International Power Electronics Conference, IPEC'95. IEEJ, April 1995, pp. 1-6.

[5] P. Vas, M. Rashed, A. Joukhadar, and C. Ng, "Sensorless induction motor and permanent magnet synchronous motor drives using natural field orientation," COMPEL: International Journal for Computation and Mathematics in Electrical and Electronic Engineering, vol. 21, no. 2, pp. 323-337, 2002.

[6] M. Kazmierkowski, M. Malinowski, D. Sobczuk, F. Blaabjerg, and J. Pedersen, "Simplified stator flux oriented control," in Proceedings of the IEEE International Symposium on Industrial Electronics, ISIE'99, vol. 2, 1999, pp. 474-479.

[7] D. Pulle, "Analysis of the nfo pm type drive," July 2001, private Report.

[8] — , "Nfo instability: defining limits, part 2," March 2001, private Report.

[9] _ "Analysis of the nfo type drive," June 2001, private Report.

[10] R. Betz, G. Mirzaeva, and D. Pulle, "Frame alignment stability issues in natural field orientation," School of Electrical Engineering and Computer Science, University of Newcastle, Australia, Tech. Rep. V1.13, January 2006. 